Original Article

Journal of Epilepsy Research

pISSN 2233-6249 / eISSN 2233-6257

\title{
Increased Expression of Tumor Necrosis Factor-Alpha in the Rat Hippocampus after Acute Homocysteine Administration
}

\author{
Seong Hee Kim, MD', Kwangsoo Kim, MD', Ji Hyun Ahn, MD², Hee Kyung Chang, MD² \\ Departments of ${ }^{1}$ Neurology and ${ }^{2}$ Pathology, Kosin University College of Medicine, Busan, Korea
}

Background and Purpose: This paper evaluated the effect of acute homocysteine administration on inflammatory cytokine tumor necrosis factor-alpha (TNF- $\alpha$ ) expression and neuronal apoptosis in the rat hippocampus and investigated the effects of vitamin $C$ treatment on homocysteine-induced inflammation and neuronal death.

Methods: Subjects were three-week-old, male Sprague-Dawley rats. Rats for the control group, we injected saline solution into the rats' abdominal cavities for one week. Rats in the second group received 1 injection of homocysteine $(11 \mathrm{mmol} / \mathrm{kg})$ into their abdominal cavities after 1 week of saline solution administration. For the third group, we injected the rats with vitamin $C(100 \mathrm{mg} / \mathrm{kg})$ for a week, followed by 1 injection of homocysteine. The hippocampi were stained with an anti-TNF- $\alpha$ antibody, and apoptosis was evaluated using the TUNEL staining method.

Received Sep. 16, 2010 Accepted Nov. 29, 2010

Corresponding author: Kwangsoo Kim Department of Neurology, Kosin University College of Medicine, 34 Amnam-dong, Seo-gu, Busan 602-703, Korea

Tel. +82-51-990-6274

Fax. +82-51-990-3077

E-mail; nekim@ns.kosinmed.or.kr
Results: The homocysteine-injected rats had strong TNF- $\alpha$ expression in every hippocampal region. Vitamin C significantly reduced TNF- $\alpha$ expression in the hippocampus's CA1 region. Acute homocysteine administration did not cause apoptosis in the hippocampus.

Conclusions: The pro-inflammatory cytokine TNF- $\alpha$ may mediate elevated homocysteine levels' contributions to inflammatory reactions, and vitamin $\mathrm{C}$ has some protective effect on inflammatory reactions in the CA1 hippocampal region. (2011;1:6-12)

Key words: Homocysteine; TNF- $\alpha$; Apoptosis; Vitamin C; Hippocampus

\section{Introduction}

Homocysteine is an amino acid generated during methionine metabolism. Remethylation converts almost half of such homocysteine to methionine; folate, vitamin $B_{2}$, and vitamin $B_{12}$ act as enzyme cofactors during this process. The remaining homocysteine is used in cysteine synthesis via transsulfuration by the vitamin $\mathrm{B}_{6}$-dependent enzyme cystathionine beta synthase. Deficiencies in folate, vitamin

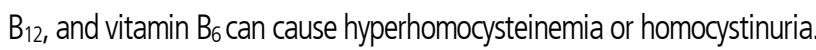
Homocysteine accelerates the growth of vessel smooth muscles, thereby inducing arteriosclerosis, and it has neurotoxic effects. High homocysteine levels are found in patients with neuropsychiatric diseases, such as stroke, Alzheimer's disease, epilepsy, depression, and post-traumatic stress syndrome [1-5].

Hyperhomocysteinemia causes neurodegeneration by means of several mechanisms, such as over-stimulation of N-methyl-Daspartate (NMDA) receptors [6] and auto-oxidation of homocysteine, which leads to the formation of superoxide and hydrogen peroxide [7]. Moreover, homocysteine potentiates glutamate and $\beta$-amyloid neurotoxicity $[8,9]$ and inhibits the expression of antioxidant enzymes [10]. Hyperhomocysteinemia induces apoptotic processes in the hippocampus via a mitochondria-mediated pathway [11]. These findings suggest that homocystinuric patients' neuronal cell death and convulsions may be due to the excitotoxicity and oxidative stress from high homocysteine concentrations [12]. Oxidative stress contributes to the pathogenesis of neurodegenerative disorders, dementia, and epilepsy [13]. The accumulation of homocysteine can induce pro-inflammatory reactions $[14,15]$. Homocysteine can increase tumor necrosis factor-alpha (TNF- $\alpha$ ) expression, which in turn upregulates nicotinamide adenine dinucleotide phosphate (NADPH) oxidase and inducible nitric oxide synthase [16]. Multiple studies have demonstrated the close link between inflammation and oxidative stress.

The water-soluble antioxidant ascorbic acid (vitamin C) protects biological macromolecules by scavenging reactive oxygen and nitrogen species [17]. Vitamin C, an exogenous antioxidant, inhibits neuronal damage from lipid peroxidation [18-20] and removes free radicals that form from homocysteine, mainly during hyperhomo- 
cysteinemia [21]. Vitamin C is an effective antioxidant and protects against homocysteine-induced oxidative stress [22].

How homocysteine triggers an inflammatory response is unknown. To evaluate whether cytokines mediate the inflammatory effects of elevated homocysteine levels, we evaluated acute homocysteine administration's effect on inflammatory cytokine TNF- $\alpha$ expression and neuronal apoptosis in the rat hippocampus. In addition, we investigated vitamin C treatment's effects on homocysteine-induced inflammation and neuronal death.

\section{Methods}

\section{Materials}

Subjects were three-week-old, male Sprague-Dawley rats, weighing $72-85 \mathrm{~g}$ and housed at a constant $22^{\circ} \mathrm{C}, 50 \%$ relative humidity, and 12-hr light/dark cycle. Each cage contained 5 or 6 rats and provided them free access to standard pelleted food and tap water. The acclimatization period was 7 days.

\section{Methods}

\section{1) Experimental treatments}

At the start of the experiment, we randomly divided 32 rats into 3 groups. For the first group, the controls (10 rats), we administered 1 week's pretreatment with daily intraperitoneal $0.9 \% \mathrm{NaCl}$. The second group (11 rats) received the same pretreatment, for 1 week, followed by 1 intraperitoneal injection of DL-homocysteine $(11 \mathrm{mmol}$ of homocysteine/kg body weight; Sigma-Aldrich Co., St. Louis, MO, USA). For the third group (11 rats), we administered 1 week's pretreatment with daily intraperitoneal L-ascorbic acid $(100 \mathrm{mg} / \mathrm{kg}$; Sigma-Aldrich Co., St. Louis, MO, USA), followed by 1 intraperitoneal injection of homocysteine $(11 \mathrm{mmol} / \mathrm{kg})$. Two hours after each rat's final injection of homocysteine or saline, we sacrificed the rat.

\section{2) Behavioral recordings}

For $90 \mathrm{~min}$ after the final homocysteine administration, we observed and recorded the rats' behaviors, specifically noting the occurrence of convulsive behaviors. We assessed this by noting the incidence of motor seizures, determining seizure severity via the rating scale of Stanojlovic et al. [23], which defines grades as follows: 1, head nodding, lower jaw twitching; 2, myoclonic body jerks, bilateral forelimb clonus with full rearing; 3 , progression to generalized clonic convulsions followed by tonic extension of foreand hind-limbs and tail; and 4, prolonged severe tonic-clonic convulsions lasting over 10 seconds or frequent repeated episodes of clonic convulsions for over 5 minutes. In addition, we recorded the latency to seizure, defined as the time from the homocysteine injection to the first seizure response.

\section{3) Tissue histology}

Brains were fixed in 10\% formalin for 24 hours and dissected through the coronal plane bregma, $-3.5 \mathrm{~mm}$ to $-3.0 \mathrm{~mm}$. We embedded the blocks in paraffin, cut them to $5-\mu m$ thickness using a microtome, mounted them on poly-L-lysine-treated slides, and air-dried them on a $60^{\circ} \mathrm{C}$ heat plate overnight. Sections were then stained with hematoxylin and eosin.

\section{4) Tumor necrosis factor-alpha (TNF- $\alpha$ ) immunohistochemistry}

To perform the TNF- $\alpha$ immunohistochemical staining, we used an automated staining system (Bond-Max, Leica Biosystems, Newcastle upon Tyne, UK), following the manufacturer's protocols. The 5- $\mu$ m-thick sections were mounted on positively charged slides, deparaffinized in xylene for 10 minutes, and rehydrated by means of graded ethyl alcohol solutions. Antigen retrieval was performed at $750 \mathrm{~W}$ for 45 minutes $(3 \times 15$ minutes) in citrate buffer $(\mathrm{pH} \mathrm{6.0)}$. Then, we transferred the sections to the Leica Bond-Max IHC staining system, using TNF- $\alpha$ antibody (polyclonal, Abbiotec, San Diego, CA, USA) as the primary antibody, at a 1:80 dilution. We determined the degree of TNF- $\alpha$ immunohistochemical staining as the percentage of positively-stained cells.

\section{5) Terminal deoxynucleotidyl transferase-mediated uridine} 5 '-triphosphate-biotin nick end labeling (TUNEL) histochemistry

Apoptosis and DNA fragmentation were detected in tissue sections using a TUNEL assay. The paraffin-embedded sections were deparaffinized, and then $3^{\prime}-\mathrm{OH}$ terminal DNA fragments were labeled, using a DNA Fragmentation Detection kit (Calbiochem, California, CA, USA). Preparations were digested in a $5 \mu \mathrm{g} / \mathrm{mL}$ solution of proteinase $K$, for 20 minutes at room temperature. To block endogenous peroxidase activity, we incubated the slides for 10 minutes with $2.0 \%$ hydrogen peroxidase in phosphate-buffered saline and then applied equilibration buffer directly to the slide for 30 minutes. Next, we incorporated digoxigenin-deoxyuridine triphosphate and working-strength terminal deoxynucleotidyl transferase in a humidified chamber at $37^{\circ} \mathrm{C}$ for 90 minutes, stopping the reaction with a 10-minute incubation in Stop/Wash buffer. Then we added the anti-digoxigenin-peroxidase conjugate and incubated the tissue sections in a humidified chamber for 20 minutes at room temperature. 
The staining was developed using diaminobenzidine $50 \mathrm{mg}$ in $10 \mu \mathrm{L}$ of $0.05 \mathrm{~mol} / \mathrm{L}$ Tris buffer (pH 7.6) and $20 \mu \mathrm{L}$ of $3 \%$ hydrogen peroxide, for 5 minutes. Finally, the preparations were counterstained with hematoxylin. We established 5 tissue groups according to the relative TUNEL staining intensity, i.e., the percentage of positive neurons, as follows: 0 , none; +1 , less than $25 \%$ positive neurons; +2 , 26-50\% positive neurons; $+3,51-75 \%$ positive neurons; +4 , more than $75 \%$ positive neurons. 8

\section{Statistical analysis}

Results were analyzed by comparing the degree of TNF- $\alpha$ expression and apoptosis in the CA1, CA2, CA3, and CA4 regions of the hippocampus between the two experimental groups and the control group. Statistical analyses were conducted using SPSS (version 12.0). We employed t-tests to evaluate the significance of the differences between experimental and control groups regarding TNF- $\alpha$ expression and degrees of apoptosis. A $p$-value $<0.05$ was considered statistically significant.

\section{Results}

\section{Homocysteine-induced behaviors and seizure patterns}

Each rat crouched and stopped moving for several minutes after its homocysteine injection. The rats then performed motions such as face-washing, smelling, dragging their legs when walking, and chewing. Most animals exhibited convulsions. Seizure patterns usually began with bending the head and neck backward, accompanied by the clonus of both forelimbs. Usually, the animal fell to either side following clonus. Then, motor seizure intensity increased, accompanied by wild running locomotion; subsequently, the animal would fall to either side, with clonic movements of all extremities. This phase was usually followed by tonic extension of the forelimbs and tonic flexion of the hindlimbs. Between the convulsions, the animal would experience akinesia. In the experimental groups, $91.7 \%, 87.5 \%, 37.5 \%$, and $8.3 \%$ of the rats experienced the $1 s t$, 2nd, 3rd, and 4th stages of convulsion, respectively. We observed no seizure behaviors in the control group.
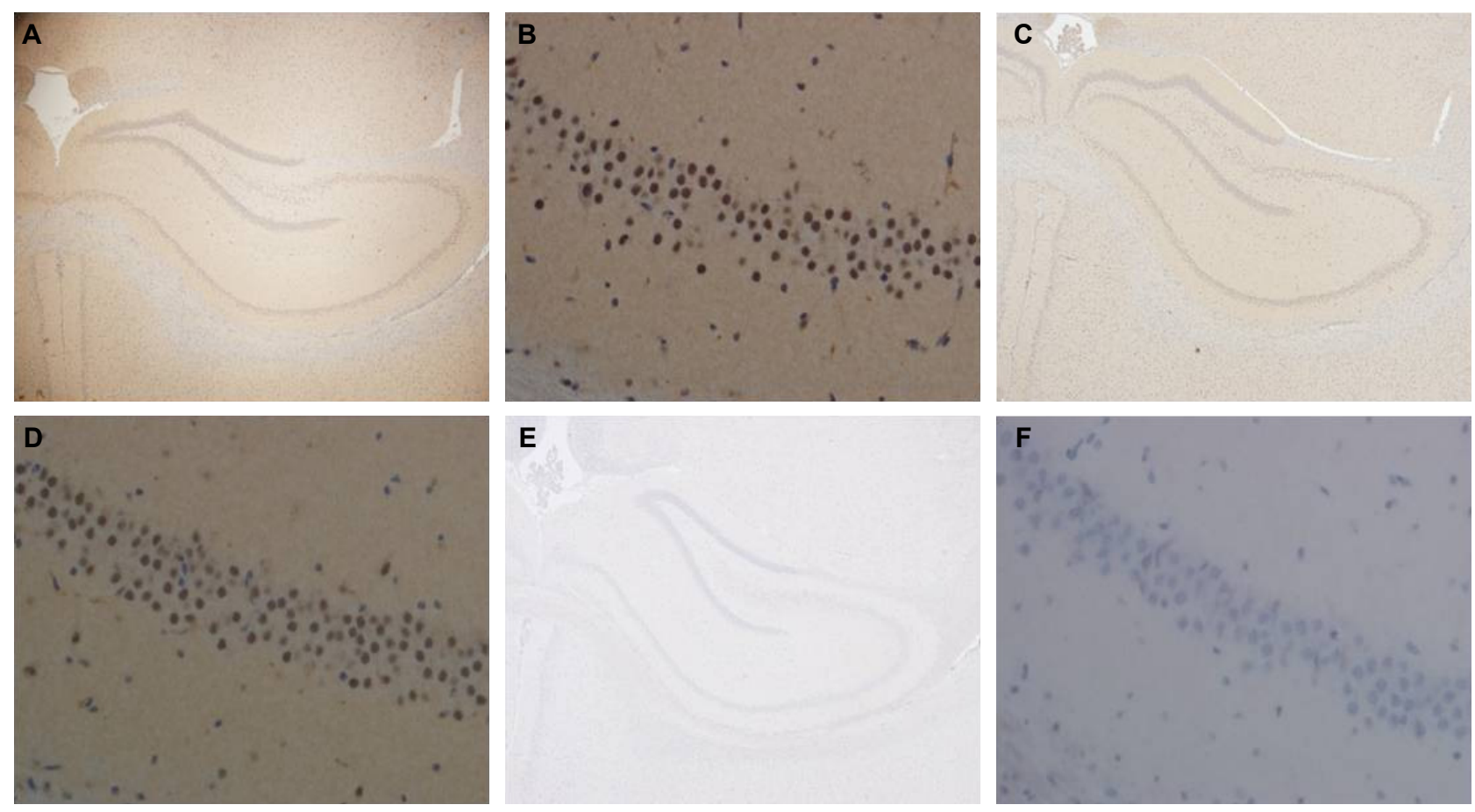

Figure 1. Immunohistochemistry for TNF- $\alpha$. (A, B) At 2 hours after homocysteine administration, strong TNF- $\alpha$ expression occurs in whole regions of the hippocampus in a saline-pretreated homocysteine-administered rat. (C, D) Strong TNF- $\alpha$ expression appears in whole regions of the hippocampus in a vitamin C-pretreated homocysteine-administered rat. (E, F) In the hippocampus of a control rat, no significant TNF- $\alpha$ expression occurs (A, C, \& E × 40; B, $D, \& F \times 400$ ). 


\section{Expression of TNF- $\alpha$ in the rat hippocampus}

Acute homocysteine administration induced strong TNF- $\alpha$ expression in all regions of the rat hippocampus (Figure 1). In the saline-pretreated homocysteine group, the percentages of positively

Table 1. Comparison of TNF- $\alpha$ expression in various rat hippocampal regions between the saline-pretreated homocysteine group (Group SH) and the vitamin C-pretreated homocysteine group (Group VCH)

\begin{tabular}{cccc}
\hline \multirow{2}{*}{ Hippocampus } & Group SH & Group VCH & \\
\cline { 2 - 3 } & Mean \pm SD (\%) & Mean \pm SD (\%) & \\
\hline CA1 & $92.9 \pm 2.9$ & $87.5 \pm 3.4$ & $<0.001$ \\
CA2 & $89.6 \pm 9.1$ & $90.4 \pm 1.5$ & 0.387 \\
CA3 & $85.6 \pm 6.1$ & $87.2 \pm 6.3$ & 0.057 \\
CA4 & $93.8 \pm 2.1$ & $90.4 \pm 4.0$ & 0.497 \\
\hline
\end{tabular}

TNF- $\alpha$, tumor necrosis factor-alpha; CA, cornu ammoni.
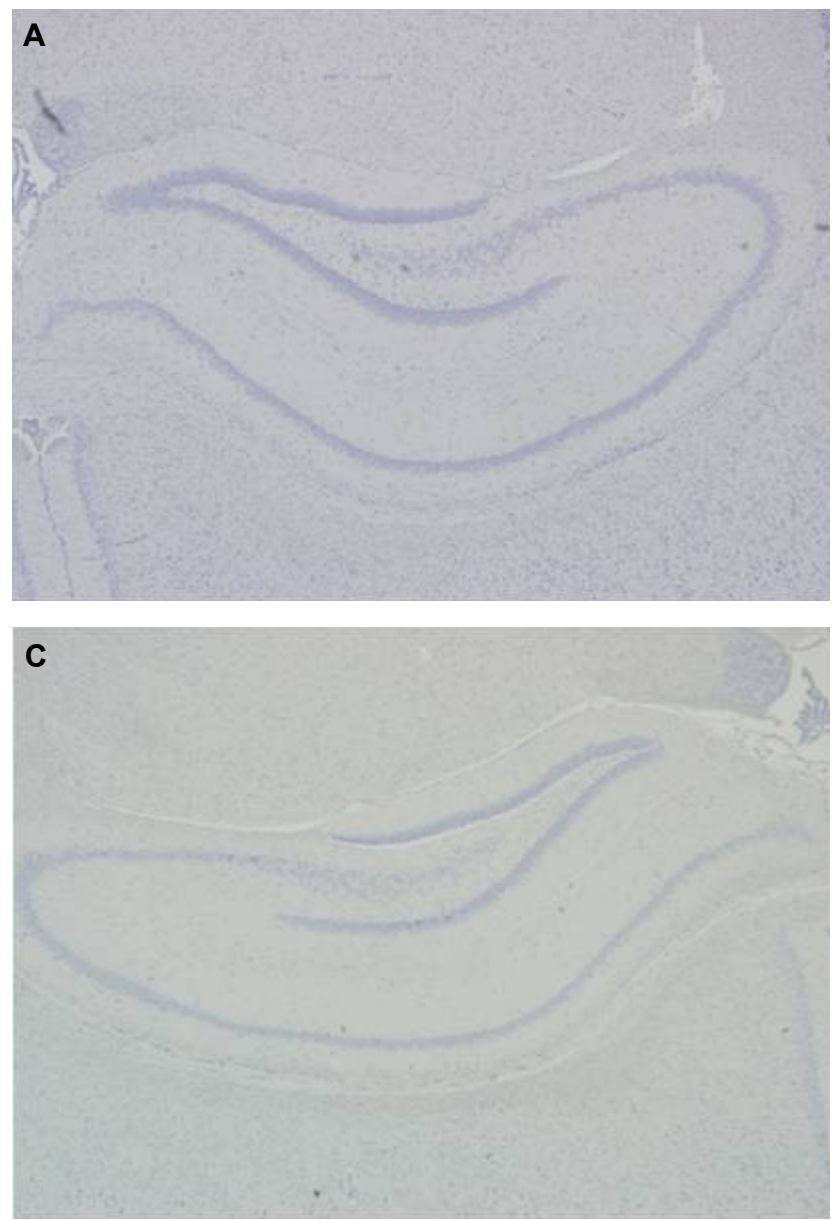

stained cells in each part of the hippocampus were as follows: $92.9 \pm$ $2.9 \%$ in CA1, 89.6 $\pm 9.1 \%$ in CA2, $85.6 \pm 6.1$ in CA3, and $93.8 \pm$ $2.1 \%$ in CA4. In the vitamin C-pretreated homocysteine group, the percentages of positively stained cells were $87.5 \pm 3.4 \%$ in CA1, $90.4 \pm 1.5 \%$ in $C A 2,87.2 \pm 6.3 \%$ in CA3, and $90.4 \pm 4.0 \%$ in CA4 When compared to the saline-pretreated homocysteine group, the vitamin C-pretreated group showed significantly reduced TNF- $\alpha$ expression in the CA1 region ( $p<0.001$; Table 1). We found no TNF$\alpha$ expression in the hippocampi of control group rats.

\section{Homocysteine-induced hippocampal neural apoptosis}

Acute homocysteine administration did not induce apoptosis in any hippocampal regions of the control or the experimental groups (Figure 2).
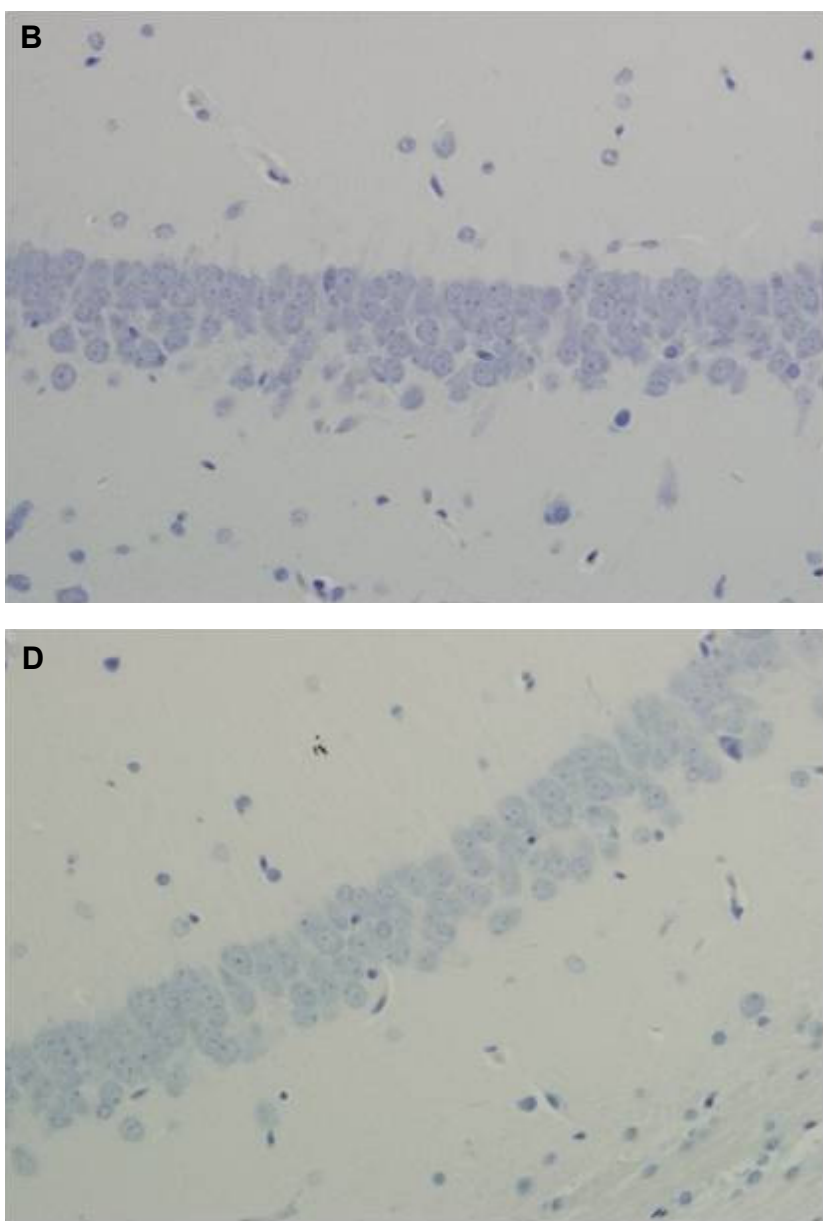

Figure 2. TUNEL histochemistry. (A, B) At 2 hours after homocysteine administration, no apoptosis appears in any whole regions of the hippocampus in a saline-pretreated homocysteine-administered rat. (C, D) In a control rat, no apoptosis appears in any whole regions of the hippocampus. (A \& C × 40; B \& $\mathrm{D} \times 400)$ 


\section{Discussion}

We found an increased TNF- $\alpha$ expression in all hippocampal regions after acute homocysteine administration. However, we observed no apoptosis in the hippocampi. Vitamin C had some protective effect against the inflammatory reactions from homocysteine in the CA1 region of the hippocampus.

Folate, vitamin B12, or vitamin B6 deficiencies can cause hyperhomocysteinemia. Auto-oxidation of homocysteine forms homocysteine, mixed disulfides, and homocysteine thiolactone as well as active oxygen groups, including the superoxide anion, hydrogen peroxide, hydroxyl radicals, and thiol free radicals. Oxidative stress causes epileptic seizures by damaging brain nerve cells. Homocysteine has an accelerating effect on the N-methyl-D-aspartate (NMDA) receptor's glutamate fusion site and an antagonist effect on the $\mathrm{N}$-methyl-D-aspartate (NMDA) receptor's glycine fusion site. Increased serum homocysteine levels occur in many neuropsychiatric diseases, such as stroke, Alzheimer's disease, epilepsy, depressive disorders, and posttraumatic stress disorder [1-3,5]. Homocysteine is a potent neurotoxic factor involved in neurodegenerative disorders $[24,25]$. Homocysteine's neurotoxic effects likely result either from the generation of reactive oxygen species due to its auto-oxidation [26] or as a consequence of NMDA receptor activation [6]. Increased homocysteine levels in cell nuclei may induce DNA strand breaks by disturbing the DNA methylation cycle [27]. Hyperhomocysteinemia induces alterations in apoptosis-related proteins and oxidative processes in neural tissue, and chronic hyperhomocysteinemia induces apoptotic processes in the hippocampus via a mitochondria-mediated pathway [11]. Homocysteine-induced neurotoxicity results in DNA damage and altered DNA repair, due to disturbances in the DNA methylation cycle, glutamate excitotoxicity via stimulation of $\mathrm{N}$-methyl-D-aspartate (NMDA) receptors, endoplasmic reticulum stress, calcium overload, and oxidative stress [28,29]. Homocysteine can increase oxidative stress by inhibiting the functioning of key antioxidant enzymes, such as extracellular superoxide dismutase [30]. Oxidative stress is likely also involved in hyperhomocysteinemiainduced apoptosis in the hippocampus. Increased oxidative stress can lead to nucleic acid, protein, and lipid damage, followed by a variety of secondary processes, such as impaired membrane enzyme activity. A cellular oxidation increase can activate redox-responsive transcription factors, such as nuclear factor-kappa B, which is a critical step in TNF- $\alpha$ gene expression [31]. TNF- $\alpha$ is a multifactorial cytokine, mainly involved in inflammatory and other immune reactions. TNF- $\alpha$, together with other proinflammatory cytokines, may play a role in the development of CNS injury. TNF- $\alpha$ plays a central role in the development of the acute-phase processes that occur in brain infarctions, as well as during CNS injury, participating in and modulating a complex neuroinflammatory network characterized by opposing neurotoxic and neuroprotective properties [32]. Bogdanski et al. [33] reported a strong positive correlation between homocysteine and TNF- $\alpha$. In our study, we showed that TNF- $\alpha$ expression increased in all regions of the hippocampus after homocysteine administration. These results indicate high homocysteine levels contribute to the inflammatory process in hippocampal nerve cells.

Homocysteine accumulates in certain brain structures, including the hippocampus, cerebellum, striatum, and subventricular zone. Blaise et al. [34] reported that homocysteine distribution in the brain was not uniform but, rather, was concentrated in specific regions, including the cerebellum's granular cell layer, the hippocampus's CA1 pyramidal layer, the caudate-putamen, and the subventricular zone lining the lateral ventricle. Chung et al. [35] reported high amounts of homocysteine in the hippocampal CA1 layer and in cerebellar nuclei. Researchers have observed homocysteine accumulation in both neuronal cells and astrocytes of rat brains [34].

Langmeier et al. [36] reported homocysteic acid induced serious alterations in the hippocampi of immature rats, which most likely reflects both homocysteic acid's direct neurotoxic effects and prolonged seizure activity. Their study found extensive damage, with features of both necrotic and apoptotic death, in CA1 and CA3 pyramidal cells and the dentate gyrus's granular cell layer [36]. Langmeier et al. [36] also reported seizure activity caused the damage to the dentate granular cells, whereas, apparently, seizures and homocysteic acid's direct toxic action both caused pyramidal cell death [36]. Mattson and Shea found cells containing homocysteine in the hippocampi of folate deficient rats were apoptotic, consistent with the observation of increased p53 expression, which is one of the major routes by which homocysteine promotes apoptotic cell death [28]. Another study found immunological labeling of apoptotic cells revealed a substantially greater number of cells undergoing apoptosis in the CA1 hippocampal layer of rats on a vitamin B-deficient diet, as compared with controls [34].

We did not observe apoptosis in the hippocampus after acute homocysteine administration. This may indicate apoptosis does not occur in the hippocampus less than 2 hours after homocysteine administration. 
Vitamin $C$ is an exogenous antioxidant and can alter seizureinduced oxidative stress and neuronal damage [20]. Vitamin C exerts potent anticonvulsant and neuroprotective effects [19] and protects against the neurotoxic effects of glutamic acid and NMDA by interacting with the NMDA receptor [37]. Pretreatment with vitamin $\mathrm{E}$ and $\mathrm{C}$ prevents homocysteine-induced oxidative stress by reducing the total radical-trapping antioxidant potential and increasing the activity of sodium-potassium adenosine triphosphatase and catalase [38]. Santos et al. [20] reported vitamin C not only significantly increased catalase activity, indicative of a possible antioxidant effect, but also decreased lipid peroxidation levels. The vitamin C-induced increase in catalase activity produced neuronal protection and also decreased lipid peroxidation, the main factor responsible for neuronal damage after seizures and pilocarpine-induced status epilepticus [20].

In summary, acute homocysteine administration caused various behavioral responses and seizures in rats and induced increased expression of TNF- $\alpha$ in all hippocampal regions. Vitamin C pretreatment significantly reduced TNF- $\alpha$ expression in the hippocampus's CA1 region. These results suggest the proinflammatory cytokine TNF- $\alpha$ may mediate high homocysteine levels' contribution to the inflammatory process. Vitamin C provided some protection against the inflammatory reaction in the CA1 region of the rat hippocampus.

\section{References}

1. Refsum H, Ueland PM, Nygard O, Vollset SE. Homocysteine and cardiovascular disease. Annu Rev Med 1998;49:31-62.

2. Bottiglieri T, Laundy M, Crellin R, Toone BK, Carney MW, Reynolds EH. Homocysteine, folate, methylation, and monoamine metabolism in depression. J Neurol Neurosurg Psychiatry 2000;69:228-32.

3. Diaz-Arrastia R. Homocysteine and neurologic disease. Arch Neurol 2000;57:1422-7.

4. Ha SW, Kim KS. Relationship between serum levels of homocysteine and folate and seizure severity in patients with idiopathic epilepsy. J Korean Epilep Soc 2008;12:22-6.

5. Levine J, Timinsky I, Vishne T, et al. Elevated serum homocysteine levels in male patients with PTSD. Depress Anxiety 2008;25:E154-7.

6. Lipton SA, Kim WK, Choi YB, et al. Neurotoxicity associated with dual action of homocysteine at the N-methyl-D-aspartate receptor. Proc Natl Acad Sci USA 1997;94:5923-8.

7. White AR, Huang $X$, Jobling MF, et al. Homocysteine potentiates copperand amyloid beta peptide-mediated toxicity in primary neuronal cultures: possible risk factors in the Alzheimer's-type neurodegenerative pathways. J Neurochem 2001;76:1509-20.
8. Folbergrova J. NMDA and not non-NMDA receptor antagonists are protective against seizures induced by homocysteine in neonatal rats. Exp Neurol 1994;130:344-50.

9. Ho P, Collins SC, Dhitavat $S$, et al. Homocysteine potentiates $\beta$-amyloid neurotoxicity: role of oxidative stress. J Neurochem 2001;78:249-53.

10. Upchurch GR Jr, Welch GN, Fabian AJ, et al. Homocysteine decreases bio-available nitric oxide by a mechanism involving glutathione peroxidase. J Biol Chem 1997;272:17012-7.

11. Baydas G, Reiter RJ, Akbulut M, Tuzcu M, Tamer S. Melatonin inhibits neural apoptosis induced by homocysteine in hippocampus of rats via inhibition of cytochrome c translocation and caspase-3 activation and by regulating pro-and anti-apoptotic protein. Neuroscience 2005;135: 879-86.

12. Grisar T. Glial and neuronal $\mathrm{Na}^{+}-\mathrm{K}^{+}$pump in epilepsy. Ann Neurol 1984;16(Suppl):128-34.

13. Halliwell B, Gutteridge JMC. Oxygen radicals in the nervous system. Trends Neurosci 1985;8:22-6.

14. Desai A, Lankford HA, Warren JS. Homocysteine augments cytokine-induced chemokine expression in human vascular smooth muscle cells: implications for atherogenesis. Inflammation 2001;25:179-86.

15. Gori AM, Corsi AM, Fedi S, et al. A proinflammatory state is associated with hyperhomocysteinemia in the elderly. Am J Clin Nutr 2005;82: 335-41.

16. Ungvari Z, Csiszar A, Edwards JG, et al. Increased superoxide production in coronary arteries in hyperhomocysteinemia: role of tumor necrosis factor-alpha, $\mathrm{NAD}(\mathrm{P}) \mathrm{H}$ oxidase, and inducible nitric oxide synthase. Arterioscler Thromb Vasc Biol 2003;23:418-24.

17. Carr A, Frei B. Does vitamin $C$ act as a pro-oxidant under physiological conditions? FASEB J 1999;13:1007-24.

18. Barros DO, Xavier SM, Barbosa CO, et al. Effects of the vitamin E in catalase activities in hippocampus after status epilepticus induced by pilocarpine in Wistar rats. Neurosci Lett 2007;420:76-9.

19. Xavier SM, Barbosa CO, Barros DO, Silva RF, Oliveira AA, Freitas RM. Vitamin $C$ antioxidant effects in hippocampus of adult Wistar rats after seizures and status epilepticus induced by pilocarpine. Neurosci Lett 2007:8:76-9.

20. Santos LFL, Freitas RLM, Xavier SML, Saldanha GB, Freitas RM. Neuroprotective actions of vitamin $\mathrm{C}$ related to decreased lipid peroxidation and increased catalase activity in adult rats after pilocarpine-induced seizures. Pharmacol Biochem Behav 2008;89:1-5.

21. Krajcovicova-Kudlackova M, Ginter E, Blazicek P, Klvanova J. Homocysteine and vitamin C. Bratisl Lek Listy 2002;103:171-3.

22. Mahfouz MM, Kummerow FA. Vitamin C or vitamin B6 supplementation prevent the oxidative stress and decrease of prostacyclin generation in homocysteinemic rats. Int J Biochem Cell Biol 2004;36:1919-32.

23. Stanojlovic O, Rasic-Markovic A, Hrncic D, et al. Two types of seizures in homocysteine thiolactone-treated adult rats, behavioral and electroencephalographic study. Cell Mol Neurobiol 2009;29:329-39.

24. Clarke R, Smith AD, Jobst KA, Refsum $H$, Sutton L, Ueland PM. Folate, 
vitamin B12, and serum total homocysteine levels in confirmed Alzheimer disease. Arch Neurol 1998;55:1449-55.

25. Seshadri S, Beiser A, Selhub J, et al. Plasma homocysteine as a risk factor for dementia and Alzheimer's disease. N Engl J Med 2002;346:476-83.

26. Dayal S, Arning E, Bottiglieri T, et al. Cerebral vascular dysfunction mediated by superoxide in hyperhomocysteinemic mice. Stroke 2004;35: 1957-62.

27. Blount $B C$, Mack MM, Wehr CM, et al. Folate deficiency causes uracil misincorporation into human DNA and chromosome breakage: implication for cancer and neuronal damage. Proc Natl Acad Sci USA 1997:94:3290-5.

28. Mattson MP, Shea TB. Folate and homocysteine metabolism in neural plasticity and neurodegerative disorders. Trends Neurosci 2003;26:137-46.

29. Ho PI, Ortiz D, Rogers E, Shea TB. Multiple aspects of homocysteine neurotoxicity: glutamate excitotoxicity, kinase hyperactivation and DNA damage. J Neurosci Res 2002;70:694-702.

30. Yamamoto $M$, Hara $H$, Adachi T. Effects of homocysteine on the binding of extracellular superoxide dismutase to the endothelial cell surface. FEBS Lett 2000;486:159-62.

31. Allen RG, Tresini M. Oxidative stress and gene regulation. Free Radic Biol Med 2000;28:463-99.

32. Intiso D, Zarralli MM, Lagioia $G$, et al. Tumor necrosis factor alpha serum levels and inflammatory response in acute ischemic stroke patients. Neurol Sci 2003;24:390-6.

33. Bogdanski P, Pupek-Musialik D, Dytfeld J, Lacinski M, Jablecka A, Jakubowski H. Plasma homocysteine is a determinant of tissue necrosis factor- $\alpha$ in hypertensive patients. Biomed Pharmacother 2008;62:360-5.

34. Blaise SA, Nedelec E, Schroeder $H$, et al. Gestational vitamin B deficiency leads to homocysteine-associated brain apoptosis and alters neurobehavioral development in rats. Am J Pathol 2007;170:667-79.

35. Chung YH, Hong JJ, Shin CM, Joo KM, Kim MJ, Cha Cl. Immunohistochemical study on the distribution of homocysteine in the central nervous system of transgenic mice expressing a human Cu/Zn SOD mutation. Brain Res 2003;967:226-34.

36. Langmeier M, Folbergrova J, Haugvicova R, Pokorny J, Mares P. Neuronal cell death in hippocampus induced by homocysteic acid in immature rats. Epilepsia 2003;44:299-304.

37. Bell JA, Beglan $C L$, London ED. Interaction of ascorbic acid with the neurotoxic effects of NMDA and sodium nitroprusside. Life Sci 1996; 58:367-71.

38. Wyse ATS, Zugno Al, Streck EL, et al. Inhibition of $\mathrm{Na}^{+}, \mathrm{K}^{+}$-ATPase activity in hippocampus of rats subjected to acute administration of homocysteine is prevented by vitamin E and C treatment. Neurochem Res 2002; 27:1685-9. 